\title{
Providing Natural Water Structure Surrounding Highly Mobile Maltose Groups Conjugated with Polyrotaxanes
}

\author{
Hiroaki Hirose, ${ }^{1}$ Haruyuki SAno, ${ }^{1, \dagger}$ Goro Mizutani, ${ }^{1,2}$ \\ Masaru EGUCHI, ${ }^{1}$ Tooru OoYA,${ }^{1,2, \dagger \dagger}$ and Nobuhiko YUI ${ }^{1,2, \dagger}$ \\ ${ }^{1}$ School of Materials Science, Japan Advanced Institute of Science and Technology (JAIST), \\ 1-1 Asahidai, Nomi 923-1292, Japan \\ ${ }^{2}$ JAIST 21st Century COE Program, 1-1 Asahidai, Nomi 923-1292, Japan
}

(Received April 20, 2006; Accepted July 12, 2006; Published August 30, 2006)

\begin{abstract}
KEY WORDS Water Chemistry / Supramolecular Chemistry / Raman Spectroscopy / Calorimetry /
\end{abstract} [doi:10.1295/polymj.PJ2006017]

Polyrotaxane (pRX) has been known as a supramolecular assembly, in which many cyclic molecules like $\alpha$-cyclodextrins ( $\alpha$-CDs) are threaded onto a linear polymeric chain like poly(ethylene glycol) (PEG) capped with bulky end-groups. Since $\alpha$-CDs in the complex can freely slide and rotate along the PEG chain, ${ }^{1}$ the $\mathrm{pRX}$ is expected to act as a nano-scaled molecular machine. We found that ligand-pRX conjugates recognize binding proteins in a multivalent manner. ${ }^{2,3}$ Recently, we measured relative potency of concanavalin A (Con A)-induced hemagglutination inhibition to assess multivalent interaction of maltose-pRX conjugates (Mal-pRXs) and Con A, and found that the higher mobility of maltose groups as well as $\alpha$-CDs in the Mal-pRXs remarkably enhances the multivalent interaction of the maltose and the binding proteins. ${ }^{4,5} \mathrm{~A}$ certain Mal-pRX that shows the highest mobility of maltose showed the largest value of the association constant and the initial rate of binding, suggesting the contribution of both the higher mobility and the multivalent interaction with Con A. ${ }^{5}$

In the above binding event of Mal-pRXs in aqueous solutions, water structures surrounding the Mal-pRXs seem to play an important role. In our previous study, ${ }^{6}$ the water structure around the Mal-pRXs has been studied from Raman spectra of the collective stretching vibration of $\mathrm{OH}$ bonds (collective band). The collective band intensity reflects the order of the network structure of water molecules in the solution. ${ }^{6}$ It was found that the order of water structure is higher as the mobility of maltose groups in the Mal-pRXs is higher. Nevertheless, the reason for this correlation was not clarified.
In the present study, the effect of maltose mobility in the Mal-pRXs on the water structure has been investigated by using a combination of differential scanning calorimetry (DSC) and Raman spectroscopy. From the DSC measurement, we can evaluate the amount of bound water in some sample solutions. ${ }^{7-9}$ Taking the results of DSC and Raman measurements into account, a mechanism of the effect of the motion of maltose groups on the hydration structure of MalpRXs has been proposed.

\section{EXPERIMENTAL}

\section{Materials}

Mal-pRXs consisting of PEG, maltose-introduced $\alpha$-CDs, and benzyloxycarbonyl-L-tyrosine as shown in Figures 1a-1c were used as sample materials. The details of the sample preparation and the chemical structure of these conjugates were described in our previous paper. ${ }^{4,5}$ In order to control the mobility of the maltose groups in the pRX, three pRX samples with 22\%-, 38\%-, 53\%-CD threading on a PEG chain $\left(M_{\mathrm{n}}=20,000\right)$ were prepared, as shown in Table I. The concentration of $\mathrm{pRX}$ in these samples was $3.33 \times 10^{-5} \mathrm{M}$. The total number of maltose groups per pRX in these samples was set to be similar values (about 240). Consequently, the maltose concentration in the three sample solutions was approximately the same $\left(\sim 8 \times 10^{-3} \mathrm{M}\right)$. As a reference sample, a maltose-poly(acrylic acid) conjugate (sample name of Mal-PAA) was prepared as shown in Table I and Figure 1d. The mobility of maltose groups in the Mal-PAA was very low due to the covalent binding

\footnotetext{
To whom correspondence should be addressed (H. Sano, Tel: +81-761-51-1522, Fax: +81-761-51-1149, E-mail: h-sano@jaist.ac.jp, N. Yui, Tel: +81-761-51-1640, Fax: +81-761-51-1645, E-mail: yui@jaist.ac.jp).

†Present address: Department of Intelligent Design System Engineering, Toyama Prefectural University, 5180 Kurokawa, Imizu 939-0398, Japan
} 
(a)

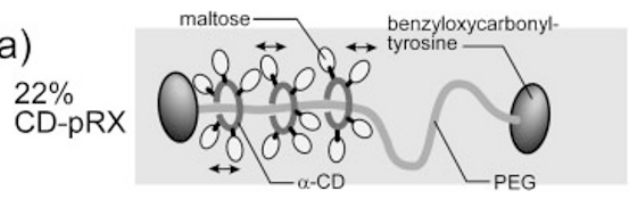

(b)

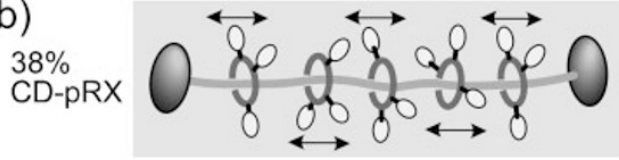

(c)

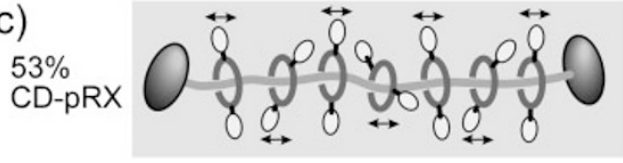

(d)

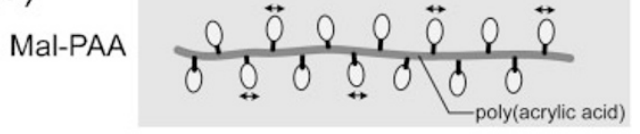

(e)

Mal

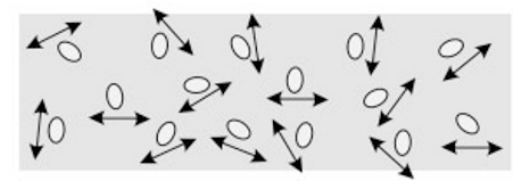

Figure 1. Molecular structure of maltose-pRX conjugates with $22 \%$ - (a), $38 \%$ - (b), $53 \%$ - (c) CD threading on a PEG chain, maltose-poly(acrylic acid) conjugate (d), and free maltose (e) in an aqueous solution. Length of arrows represents the image of maltose mobility.

of maltose groups onto the PAA backbone. ${ }^{5}$ The Mal-PAA exhibits the similar number of maltose and carboxyl groups. As another reference sample with higher mobility of maltose groups, free maltose solution (sample name of Mal) was also prepared, as shown in Table I and Figure 1e. The maltose concentration in these sample solutions was approximately the same $\left(\sim 8 \times 10^{-3} \mathrm{M}\right)$. Therefore, by comparing these samples, the influence of the dynamic motion of maltose groups on the surrounding water molecules can be assessed under the same condition. All the samples were dissolved in a $0.1 \mathrm{M}$ phosphate-buffered saline (PBS) (pH 7.4) containing $0.1 \mathrm{mM} \mathrm{CaCl}_{2}$ and $0.1 \mathrm{mM} \mathrm{MnCl}$.

The mobility of maltose groups in the solution was estimated by measuring the relaxation time $T_{2}$ of $\mathrm{C}(1) \mathrm{H}$ of maltose groups in the NMR experiment. ${ }^{4,5}$

\section{Raman Measurement}

Raman spectra of the samples in glass cells were measured in $90^{\circ}$ scattering geometry. The incident light was the $632.8 \mathrm{~nm}$ line of a He-Ne laser, and the power was $7 \mathrm{~mW}$. The measured spectral range was from 2500 to $4000 \mathrm{~cm}^{-1}$, covering the $\mathrm{OH}$ stretching mode frequencies. The polarization combinations were such that the exciting and the scattered polarizations were parallel or perpendicular to each other. All the measurements were performed at room temperature. The details of the Raman measurement have been described in our previous paper. ${ }^{6,10}$

The intensity of the collective band $\left(I_{C}\right)$, attributable to the synchronous stretching vibration of $\mathrm{OH}$ bonds in the network structure of water, can be obtained from a difference between the integrated Raman scattering intensities in the parallel and perpendicular polarization configurations $\left(I_{\|}\right.$and $\left.I_{\perp}\right): 6,10,11$

$$
I_{C}(\omega)=I_{\|}(\omega)-I_{\perp}(\omega) / \rho_{O H},
$$

where $\rho_{O H}$ is the depolarization ratio of the noncollective band. Furthermore, it is normalized as

$$
C_{X}=\int I_{C}(\omega) d \omega / \int I_{\|}(\omega) d \omega
$$

$C_{X}$ can be used as an indicator of the order of the structure of water molecules. For example, when the order of bulk water clusters is broken by introducing solute molecules to an aqueous solution, $C_{X}$ becomes small.

Since the sample solutions used in this study include maltose-introduced $\alpha$-CDs (Mal- $\alpha$-CDs) having many $\mathrm{OH}$ groups, one may point out that Raman signal of the $\mathrm{OH}$ groups in the Mal- $\alpha$-CDs disturbed the precise analysis of the collective band for water. However, the number of $\mathrm{OH}$ groups of the Mal- $\alpha$ CDs is estimated to be $\sim 0.2 \%$ of the total number of $\mathrm{OH}$ groups in the sample solutions. Therefore, Raman signal of the Mal- $\alpha$-CDs is negligible for the present analysis.

\begin{tabular}{|c|c|c|c|c|c|c|}
\hline Sample name & No. of $\alpha-\mathrm{CD}$ & $\begin{array}{c}\alpha-C D \\
\text { threading } \\
(\%)^{b}\end{array}$ & $\begin{array}{c}\text { No. of maltose } \\
\text { groups per } \\
\alpha-\mathrm{CD}\end{array}$ & $\begin{array}{l}\text { No. of maltose } \\
\text { groups }\end{array}$ & $\begin{array}{l}\text { Concentration } \\
\text { of } \alpha \text {-CD } \\
\left(\times 10^{-3} \mathrm{M}\right)\end{array}$ & $\begin{array}{l}\text { Concentration of } \\
\text { maltose groups } \\
\quad\left(\times 10^{-3} \mathrm{M}\right)\end{array}$ \\
\hline $22 \%$ CD-pRX ${ }^{a}$ & 50 & 22 & 4.6 & 230 & 1.66 & 7.65 \\
\hline $38 \%$ CD-pRX ${ }^{a}$ & 85 & 38 & 2.9 & 244 & 2.83 & 8.12 \\
\hline $53 \%$ CD-pRX ${ }^{a}$ & 120 & 53 & 2.0 & 240 & 3.99 & 7.98 \\
\hline Mal-PAA $^{\mathrm{a}}$ & - & - & - & 240 & - & 7.98 \\
\hline Mal & - & - & - & - & - & 7.98 \\
\hline
\end{tabular}

Table I. Samples for DSC and Raman measurements

${ }^{\mathrm{a}}$ Concentration is $3.33 \times 10^{-5} \mathrm{M} .{ }^{\mathrm{b}} \alpha$-CD threading $(\%)=[$ No. of $\alpha-\mathrm{CD}] /[$ Stoichiometric no. of $\alpha-\mathrm{CD}] \times 100($ see ref 4 and 5). 


\section{DSC Analysis}

DSC thermograms of all the samples were measured by a heat flux DSC (DSC120, Seiko Co., Japan). A sample aqueous solution was put into an aluminum pan and was hermetically sealed. The weight of the sample solution for the DSC measurement was 11$16 \mathrm{mg}$, and no weight loss was observed during the measurement. The sample was cooled to $-25^{\circ} \mathrm{C}$, and then it was heated to $25^{\circ} \mathrm{C}$ with a heating rate of $2.5^{\circ} \mathrm{C} / \mathrm{min}$.

In general, bound water formed around hydrophilic groups in an aqueous solution freezes at a temperature far below $0^{\circ} \mathrm{C}$. On the other hand, free water located far from hydrophilic groups is not affected by hydrophilic groups, and it freezes near $0{ }^{\circ} \mathrm{C}$. In the measured DSC thermograms in the range from -25 to $25^{\circ} \mathrm{C}$, only one peak of heat of fusion was seen near $0^{\circ} \mathrm{C}$ for all the samples. Thus, in the present study, water showing a peak of heat of fusion near $0{ }^{\circ} \mathrm{C}$ is defined as the free water, and all the other water, not freezing in the range from -25 to $0{ }^{\circ} \mathrm{C}$, is defined as the bound water.

From the DSC thermograms observed near $0{ }^{\circ} \mathrm{C}$, the enthalpy of fusion of free water $\left(\Delta H_{f}(\mathrm{~J} / \mathrm{g})\right)$ in the sample solution was directly obtained. The amount of free water $\left(W_{f}(\mathrm{~g})\right)$ is estimated from the formula ${ }^{9}$

$$
W_{f}=\Delta H_{f} / \Delta H_{\text {Buffer }} \times W_{S},
$$

where $\Delta H_{\text {Buffer }}(\mathrm{J} / \mathrm{g})$ is the enthalpy of fusion of buffer solution, and $W_{S}(\mathrm{~g})$ is the amount of the solution. The amount of bound water $\left(W_{b}\right)$ is given by

$$
W_{b}=W_{t}-W_{f},
$$

where $W_{t}$ is the total amount of water in the solution. From eqs 3 and 4, the number of bound water molecules per one maltose group in the solution was calculated, assuming that an increase in the bound water content is attributed to maltose groups.

Raman and DSC measurements were performed three to five times for each sample solution in order to confirm the experimental reproducibility.

\section{RESULTS AND DISCUSSION}

Figure 2a shows the number of bound water molecules per one maltose group as a function of the relaxation time $T_{2}$ of $\mathrm{C}(1) \mathrm{H}$ of the maltose groups. It is known in the NMR measurement that higher mobility of molecules causes longer relaxation time $T_{2}$ due to the motional narrowing effect. ${ }^{12}$ Therefore, in Figure $2 \mathrm{a}$, one can see a positive correlation between the number of bound water molecules and the mobility of maltose groups.

Figure $2 \mathrm{~b}$ shows the normalized Raman intensity of the collective band of the sample solutions as a func-

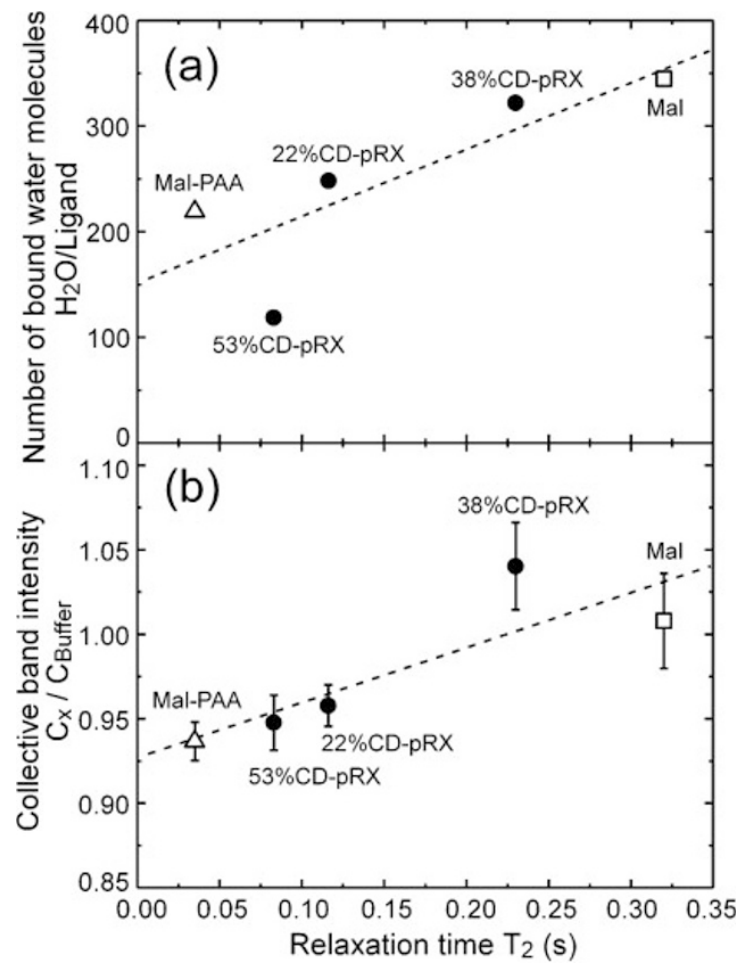

Figure 2. (a) Number of bound water molecules per one maltose group in the solutions and (b) normalized Raman intensity $\left(C_{x} / C_{\text {Buffer }}\right)$ of collective band of $\mathrm{OH}$ stretching vibration for solutions as a function of $T_{2}$-relaxation time of $\mathrm{C}(1) \mathrm{H}$ of maltose groups.

tion of the relaxation time $T_{2}$. The collective band intensity of free maltose is almost equal to that of the buffer solution $\left(C_{x} / C_{\text {Buffer }}=1.00\right)$. This result indicates that free maltose preserves the water network structure in the solution. In Figure $2 b$, a positive correlation between the collective band intensity and the relaxation time $T_{2}$ was clearly observed. Therefore, this result indicates that the degree of the order of the water clusters is higher as the mobility of maltose groups is higher in the sample solutions.

In the three Mal-pRX samples, the number of maltose groups per pRX is approximately the same, but the concentration of $\alpha-\mathrm{CD}$ is significantly different. Thus, the question may arise as to the effect of the $\alpha$-CD concentration on the collective band intensity. In our previous study, ${ }^{6}$ the collective band intensities of three maltose-introduced $\alpha$-CD solutions with the same $\alpha$-CD concentration of $22 \%, 38 \%, 53 \% \mathrm{CD}-$ pRX samples were measured. The result indicated that the collective band intensity linearly decreased with increasing the $\alpha$-CD concentration (data not shown). This behavior was quite different from the $\alpha$-CD concentration dependence of the collective band intensity of the three Mal-pRX samples. ${ }^{6}$ Therefore, $\alpha$-CDs in Mal-pRX have no significant influence to the order of the surrounding water molecules. 


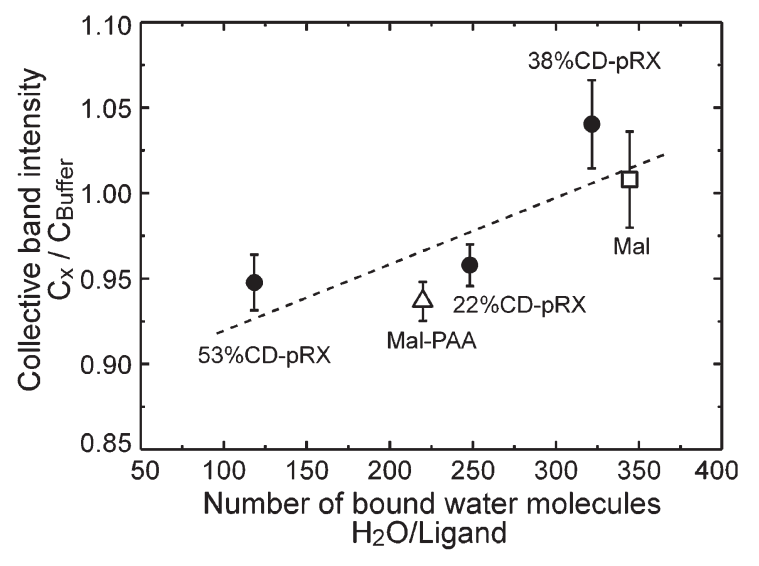

Figure 3. The relationship between number of bound water molecules per one maltose group and normalized Raman intensity of the collective band.

From Figures $2 \mathrm{a}$ and $2 \mathrm{~b}$, a relationship between the number of bound water molecules and the collective band intensity of the solutions was obtained as shown in Figure 3. It was found that the degree of the order of bulk water clusters in the solutions became higher with increasing the amount of bound water molecules. It should be noted that both the amount of bound water and collective band intensity of 38\%CD-pRX are similar to those of free maltose. This means that the water structure around maltose groups of $38 \% \mathrm{CD}$ $\mathrm{pRX}$ is similar to that of free maltose.

One may find a contradiction in the experimental results of Figures $2 a$ and $2 b$. Namely, Figure $2 b$ indicates that the order of the bulk water network structure is lower as the mobility of maltose groups is lower, and this result may be interpreted as bound water with the different structure from bulk water cluster becomes more stable with decreasing the mobility of maltose groups. In this case, one can easily imagine that the amount of bound water increases with decreasing the mobility of maltose groups. However, this prediction is not supported by the result of Figure 2a. This contradiction results from incorrect assumption of hydration structure of maltose groups. Referring to previous studies of the hydration structure, we suggest reasonable interpretation of our experimental results. So far, hydration of several saccharides in an aqueous solution has been studied by several groups. ${ }^{8,13,14}$ These studies revealed the following results. Hydroxyl groups of saccharides interact strongly with water molecules and exhibit a structure-making effect on their surrounding water. Bulk water partially has the tridymite structure as shown in Figure 4a. Since some saccharides including maltose with equatorial $\mathrm{OH}$ groups fit the tridymite structure of bulk water molecules, the tridymite structure of water around the saccharides might not be broken. For example, Figure $4 \mathrm{~b}$ shows that $\mathrm{OH}$ groups of (a)

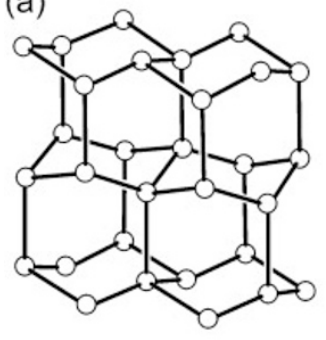

(b)

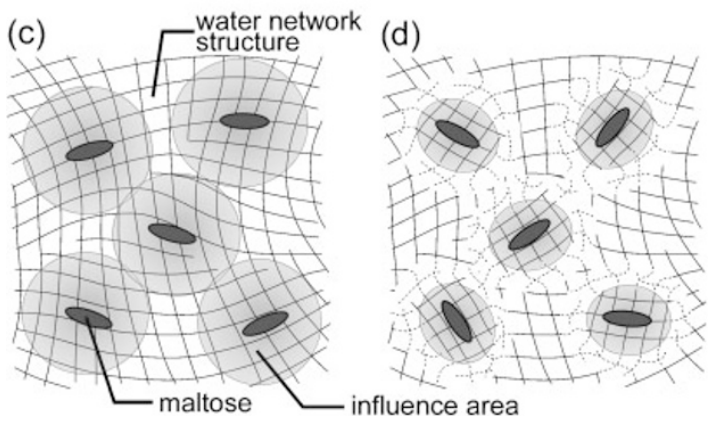

Figure 4. (a) Tridymite structure of water molecules, (b) Dglucopyranose unit with equatorial $\mathrm{OH}$ groups fitting the tridymite structure, and the water network structure including maltose groups (c) well fitting and (d) not fitting the tridymite structure of surrounding water. Open circles in (a) and (b) represent oxygen atoms and gray circle in (b) represent C-6 carbon atom.

D-glucopyranose, i.e., constituent unit of maltose, fit the tridymite structure well. The amount of bound water (unfrozen water) around a saccharide increases when the $\mathrm{OH}$ groups in the saccharide easily fit into the surrounding water structure.

Based on the above knowledge about the hydration of saccharides, we suggest the following interpretation of the results shown in Figures 2 and 3. Since free maltose and maltose groups in 38\%CD-pRX solutions show high mobility, it is easy to fit the tridymite structure of surrounding water well as shown in Figure 4c. Therefore, their influence on water should extend over a long distance. Consequently, the maltose groups have large amount of bound water, and can preserve the structure of bulk water clusters. On the other hand, when the mobility of maltose groups becomes low as in 53\%CD-pRX and Mal-PAA solutions, they cannot fit the surrounding water structure well as shown in Figure $4 \mathrm{~d}$, and their influence on water may be localized. In this case, the amount of bound water around maltose groups decreases, while network structure of water molecules in the immediate outer side of the bound water region is probably disordered due to the misfit of the structure between bound water and bulk water cluster. Therefore, the highly mobile motion of maltose groups accompanied with sliding $\alpha$ CDs in $38 \% \mathrm{CD}-\mathrm{pRX}$ maintains similar environment to free maltose in the buffer, which can provide a suitable condition for maltose recognition to the binding site of concanavalin $\mathrm{A}$, as we reported previously. ${ }^{4,5}$ 


\section{CONCLUSIONS}

The intensity of the collective band of water molecules and the amount of bound water in aqueous solutions were measured by Raman spectroscopy and DSC for maltose-polyrotaxane conjugates with different $\alpha$-CD threading. It was found that the maltose groups with higher mobility have larger number of bound water molecules in the solution and preserves the network structure of bulk water. It is suggested that high mobility of maltose groups plays an important role of the structure-making effect of maltose groups on their surrounding water.

Acknowledgment. This study was financially supported by JAIST Research Grant (grant for inhouse research projects), a Grant-in-Aid for Scientific Research (B) (No. 14380397), a program for the "Supporting young researchers with fixed-term appointments" in Special Coordination Funds for Promoting Science and Technology, from the Ministry of Education, Science, Sports and Culture, Japan, and the 21st Century COE program "Technology Creation Based on Knowledge Science”, JAIST.

\section{REFERENCES}

1. M. Ceccato, P. L. Nostro, and P. Baglioni, Langmuir, 13,
2436 (1997).

2. T. Ooya and N. Yui, J. Controlled Release, 80, 219 (2002).

3. N. Yui, T. Ooya, T. Kawashima, Y. Saito, I. Tamai, Y. Sai, and A. Tsuji, Bioconjugate Chem., 13, 582 (2002).

4. T. Ooya, M. Eguchi, and N. Yui, J. Am. Chem. Soc., 125, 13016 (2003).

5. T. Ooya, H. Utsunomiya, M. Eguchi, and N. Yui, Bioconjugate Chem., 16, 62 (2005).

6. H. Hirose, H. Sano, G. Mizutani, M. Eguchi, T. Ooya, and N. Yui, Langmuir, 20, 2852 (2004).

7. M. Tanaka, M. Tadano, N. Ishii, K. Shimura, M. Onishi, A. Mochizuki, and T. Hatakeyama, Polym. Int., 49, 1709 (2000).

8. H. Kawai, M. Sakurai, Y. Inoue, R. Chujou, and S. Kobayashi, Cryobiology, 29, 599 (1992).

9. N. Yui, K. Kataoka, A. Yamada, and Y. Sakurai, J. Controlled Release, 6, 329 (1987).

10. H. Sano, T. Ichi, Y. Kumashiro, K. Kontani, T. Kuze, G. Mizutani, T. Ooya, and N. Yui, Spectrochim. Acta, Part A, 59, 285 (2003).

11. Y. Maeda and H. Kitano, Spectrochim. Acta, Part A, 51, 2433 (1995).

12. N. Bloembergen, E. M. Purcell, and R. V. Pound, Phys. Rev., 73, 679 (1948).

13. H. Uedaira and M. Ikura, Bull. Chem. Soc. Jpn., 62, 1 (1989).

14. H. Uedaira, M. Ishimura, and S. Tsuda, Bull. Chem. Soc. Jpn., 63, 3376 (1990). 